\title{
Glicosaminoglicanos Sulfatados da Vagina e da Pele do Perineo de Mulheres no Menacme e na Pós-Menopausa, com ou sem Prolapso Genital
}

\author{
Sulfated Glycosaminoglycans of the Vagina and Perineal Skin of Women in Menacme and After \\ Menopause, with or without Genital Prolapse
}

\author{
Autor: Leonardo Robson Pinheiro Sobreira Bezerra \\ Orientador: Prof. Dr. Manoel J.B.C. Girão \\ Co-orientadores: \\ Prof $^{a}$ Dr $^{a}$ Helena B. Nader \\ Prof $^{a}$ Dr $^{a}$ Marair Gracio F. Sartori
}

Tese apresentada à Universidade Federal de São Paulo - Escola Paulista de Medicina, para a obtenção do Título de Mestre em Ginecologia, em 5/12/2002.

\begin{abstract}
Objetivo: Analisar os glicosaminoglicanos sulfatados (GAGs) na parede vaginal posterior e na pele do períneo de mulheres no menacme e na pós-menopausa, de acordo com o estágio do prolapso genital.

Métodos: 40 pacientes foram divididas em quatro grupos. Grupo A: 10 mulheres no menacme com prolapso genital estágio 1; Grupo B: 10 mulheres na pré-menopausa com prolapso genital estágios 2 e 3; Grupo C: 7 mulheres na pós-menopausa com prolapso genital estágio 1; Grupo D: 12 mulheres na pós-menopausa com prolapso genital estágios 2 e 3 . As pacientes submeteram-se à cirurgia vaginal, quando se retiraram as amostras. Os GAGs condroitim sulfato (CS), dermatam sulfato (DS) e heparam sulfato (HS) foram extraidos por proteólise, determinados por densitometria após migração eletroforética em gel de agarose.

Resultados: Dentre os GAGs encontrados o DS teve maior expressão. CS e HS foram encontrados em quantidade significativamente inferior ao DS, não havendo diferença significativa entre os seus valores dentro de cada grupo estudado. No tecido vaginal foi observada diminuição estatisticamente significante da quantidade de GAGs totais, CS, DS, HS das pacientes na pós-
\end{abstract}

menopausa em relação às no menacme. Observou-se diminuição estatisticamente significante para os valores de GAGs totais, CS, DS, HS entre as pacientes na pós-menopausa com prolapso estágios 2 e 3 em relação às no menacme independente do estágio. Observamos significante diminuição nos valores de GAGs totais, DS e HS entre as mulheres com prolapso na pós-menopausa estágios 2 e 3 em relação às na pós-menopausa no estágio 1 . Na pele do períneo a quantidade de CS e HS foi significativamente inferior nas pacientes na pós-menopausa em relação às no menacme. Existe diminuição significante da quantidade de CS entre pacientes na pós-menopausa com prolapso estágios 2 e 3 e com estágio 1 em relação às no menacme com estágio 1 .

Conclusões: Existem diferenças quantitativas e qualitativas nos glicosaminoglicanos sulfatados da matriz extracelular na mucosa vaginal e pele do períneo das pacientes no menacme e na pós-menopausa. Após a menopausa, o tecido vaginal possui menor quantidade de GAGs totais, CS, DS e HS, principalmente nas mulheres com prolapso genital estágios 2 e 3 .

RBCO 25(9): 694-695,2003

Resumo de Tese

\section{Proposta de Escore Preditivo de Recorrência em Pacientes Submetidas a Tratamento Cirúrgico Radical do Carcinoma do Colo do Útero Estádios IB e IIA}

\author{
Proposal of a Predietive Recurrence Score in Patients Submitted to Radical Surgical Treatment of \\ Stage IB and II Carcinoma of the Cervix Uteri
}

Autor: José Humberto Tavares Guerreiro Fregnani

Orientador: Dra. Maria do Rosário Dias de Oliveira Latorre

Co-orientador: Dr. Fernando Augusto Soares

Data de apresentação: 16/12/2002 (Hospital do Câncer A. C. Camargo)

Pós-Graduação da Fundação Antônio Prudente - Hospital do Câncer A. C. Camargo 